\title{
Multifrequency Monitoring of the Blazar PKS 2005-489
}

\author{
Travis Rector $^{1}$, Eric Perlman ${ }^{2}$, Rita Sambruna ${ }^{3}$, Greg Madejski ${ }^{4}$, \\ Frederik Rantakyro ${ }^{5}$ and CANGAROO collaboration ${ }^{6}$ \\ ${ }^{1}$ National Optical Astronomy Observatory, 950 N. Cherry Ave, Tucson, AZ, USA, 85719 \\ rector@noao.edu \\ ${ }^{2}$ University of Maryland BC, 1000 Hilltop Circle, Baltimore, MD, USA, 21250 \\ perlman@jca.umbc.edu \\ ${ }^{3}$ George Mason University, Department of Physics \& Astronomy, \\ 4400 University Drive, Fairfax, Virginia, USA 22030-4444 \\ rms@physics.gmu.edu \\ ${ }^{4}$ Stanford Linear Accelerator Center, 2575 Sand Hill Road, Menlo Park, CA 94025 \\ madejski@slac.stanford.edu \\ ${ }^{5}$ European Southern Observatory, Alonso de Cordova 3107, Vitacura, Santiago, Chile \\ frantaky@eso.org \\ ${ }^{6}$ Contact: Hidetoshi Kubo, Kyoto University, Department of Physics, \\ Kyoto University, Sakyo-ku, Kyoto, Japan 606-8502 \\ kubo@cr.scphys.kyoto-u.ac.jp
}

\begin{abstract}
We present a multifrequency monitoring campaign of PKS 2005-489, a bright and highly variable blazar. Simultaneous observations were completed over a 13 day period in the X-ray with RXTE, in the optical at the CTIO $0.9 \mathrm{~m}$ telescope, at TeV energies with CANGAROO-II, and at submillimetre wavelengths with SEST. Previous multiwavelength monitoring campaigns of PKS 2005-489 and other blazars have found complex flux and spectral variability behaviour, with different modes and timescales from days to hours and shorter.

PKS 2005-489 was observed in transition from a quiescent state into the early stages of a flare. A gradual increase in flux density was observed at optical and X-ray wavelengths during 19-27 August 2000, and a dramatic increase in X-ray emission was observed starting on 2 September 2000. Intraday and spectral variability were not detected during the campaign.
\end{abstract}

Keywords: BL Lacertae objects: individual (PKS 2005-489)

\section{Description of the Campaign}

The observations were designed to search for variability on both long and short timescales. The aim was that this would enable us to follow up on the unique behaviour of PKS 2005-489 during 1998. In September, there was a general increase in X-ray flux over a 3-day period, with smaller oscillations on timescales of several hours (Sambruna 2000). In October-December there was a massive flare, which peaked at $\mathrm{F}(2-10 \mathrm{keV})=3.33$ $\times 10^{-10} \mathrm{erg} \mathrm{cm}^{-2} \mathrm{~s}^{-1}$. During the flare, no variability was detected on timescales $<1$ day, and there was very little shift in $v_{\text {peak }}$ (Perlman et al. 1999; Tagliaferri et al. 2001) at $\sim 10^{15.5}-10^{16} \mathrm{~Hz}$.

We therefore designed a campaign with the following schedule: short $(\sim 2-3$ orbit) observations were taken at intervals of $\sim 0.5-1$ day during 19-26 August and 3-4 September 2000. Nearly continuous RXTE monitoring was completed during 27 August-2 September 2000. Optical monitoring with the CTIO $0.9 \mathrm{~m}$ telescope was completed during 22-28 August. PKS 2005-489 was also observed in the submillimetre by the Swedish-ESO Submillimetre Telescope (SEST) and at TeV energies with CANGAROO-II.

\section{Observations}

\section{$2.1 X$-ray Observations}

Observations were obtained with the RXTE PCU0 and PCU1 detectors. Unfortunately, the data from PCU0 are dominated by problems with bad background subtraction, making half of our data useless.

The object was much fainter than in 1998, averaging $\mathrm{F}(2-10 \mathrm{keV})=1.08 \times 10^{-11} \mathrm{erg} \mathrm{cm}^{-2} \mathrm{~s}^{-1}$. This is faint for RXTE, so the X-ray error bars are large. Only very modest X-ray variability was seen, with an increase of nearly a factor of 2 during 19-27 August and a decrease of $\sim 40 \%$ on 28 August (Figure 1). There is no evidence for variability during 29 August -1 September, however a rapid increase of $\sim 50 \%$ was observed on 2 September, which accelerated on 3-4 September. No evidence of $\mathrm{X}$-ray spectral variability was seen during the campaign. All figures are quoted for the $2-6 \mathrm{keV}$ band only, as the error bars are much larger in the hard band. Importantly, due to poor sampling, we cannot rule out the possibility of shorter timescale, chaotic variability during 19-27 August or 3-4 September.

The X-ray spectrum was fitted with a power law with $\alpha=2.11 \pm 0.08$ (90\% confidence error bar). The spectrum 
PKS2005-489 lightcurve, August 2000, 10000 s bins

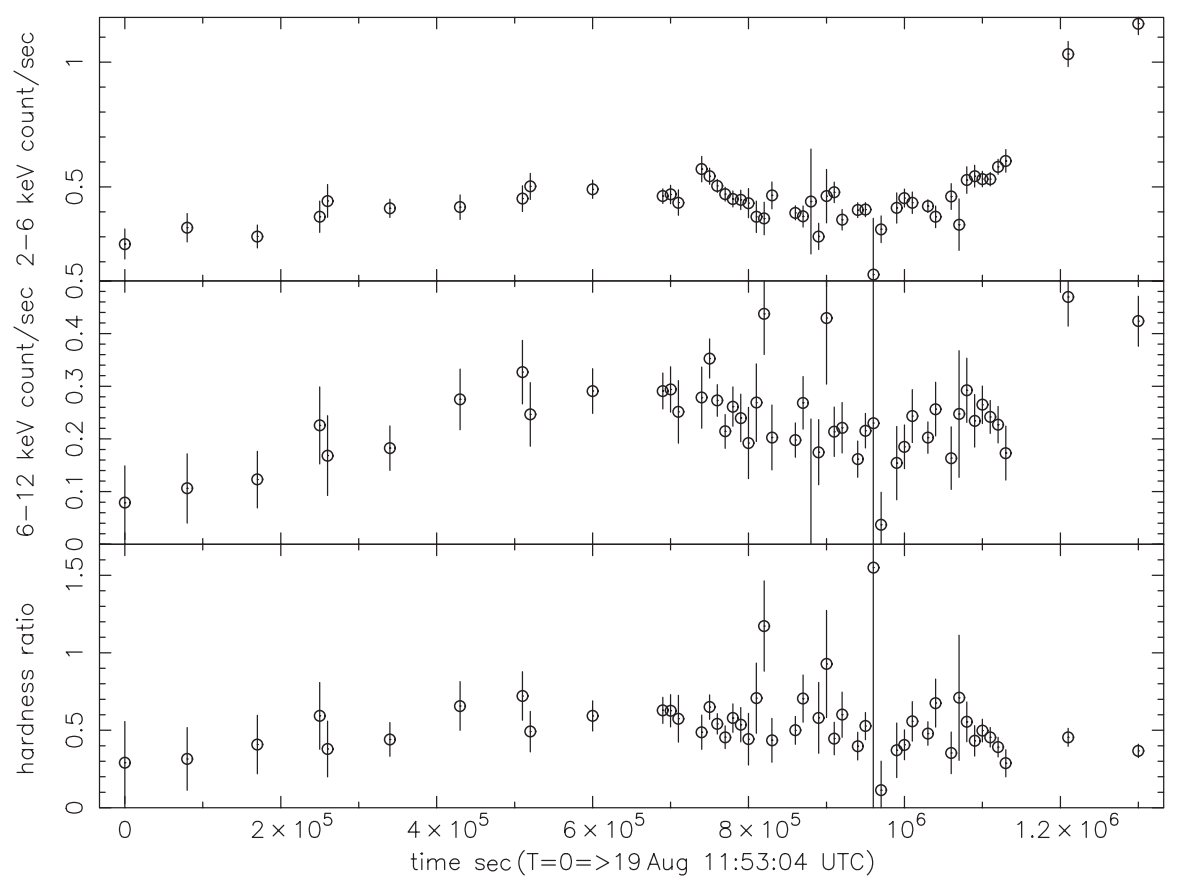

Figure 1 The RXTE lightcurve, binned to 10000 s ( 2 orbits).

was steeper than in 1998, when $\alpha=1.3-1.8$ was measured, and much more similar to the ROSAT low-state observations (Sambruna et al. 1995), where $\alpha=2$ was measured.

\subsection{Optical Observations}

Optical monitoring was completed with the CTIO $0.9 \mathrm{~m}$ telescope during 22-28 August. Optical monitoring was also scheduled in Adelaide and in Potchefstroom but unfortunately both locations were clouded out, thereby leaving $\sim 16$ hour holes per day in the optical coverage. Figure 2 shows the optical variability of PKS 2005-489. Only very modest optical variability was seen, in agreement with the quiescent state observed with RXTE: the optical flux increased linearly $\sim 13 \%$ over $\sim 400 \mathrm{ksec}$ starting 22 August. No variability is seen during 28 August. There is no evidence of colour variability, nor evidence of variability on short timescales ( $\leq 1$ day). The measured flux densities were corrected for Galactic reddening and the flux from the host galaxy (Urry et al. 2000) was subtracted.

\subsection{Submillimetre Observations}

SEST observed PKS 2005-489 on 22 August, however multiple observations were not obtained during the campaign. Flux densities of $1.34 \pm 0.40 \mathrm{Jy}$ at $150 \mathrm{GHz}$ and $1.28 \pm 0.18 \mathrm{Jy}$ at $90 \mathrm{GHz}$ were observed, indicating variability as compared to the flux densities of $1.97 \pm 0.66 \mathrm{Jy}$ at $150 \mathrm{GHz}$ and $2.13 \pm 0.39 \mathrm{Jy}$ at $90 \mathrm{GHz}$ observed on 4 March 2000.

\subsection{Gamma-ray Observations}

CANGAROO-II observed PKS 2005-489 on 28 and 30 August, and 1-2 September. Observing on 29 August and 3 September was not possible because of poor weather. These and other CANGAROO-II observations are presented by Nishijima (2002). The total on-source time for CANGAROO-II was 442 min, with 276 min on a background field. The source was not detected, with an upper limit of $\mathrm{F}(>450 \mathrm{GeV})<1.1 \times 10^{-11}$ photons $\mathrm{cm}^{-2} \mathrm{~s}^{-1}$.

\section{Multiwavelength Spectrum}

We used the average RXTE flux and spectrum, along with the average dereddened optical fluxes and SEST fluxes from 22 September, to plot the object's spectral energy distribution. We fitted the SED with a Kardashev-Pacholczyk synchrotron model (Kardashev 1962; Pacholczyk 1970). We find $v_{\text {peak }} \approx 5 \times 10^{15} \mathrm{~Hz}$, similar to the peak observed near the 1998 flare maximum (Tagliaferri et al. 2001), and also close to the value found by Sambruna et al. (1995) from noncontemporaneous data. Thus, in PKS 2005-489 the peak frequency is quite insensitive to the overall flux state. This behaviour is very different to what is found in the best-observed high-energy BL Lacs, i.e. Mkn 421 and 501 (Kataoka et al. 2001), where much higher values of $v_{\text {peak }}$ are seen during bright states.

\section{Acknowledgments}

RXTE data products were provided by the ASM/RXTE teams at MIT and at the RXTE SOF and GOF at NASA's GSFC. CTIO is part of the National Optical Astronomy Observatory, which is operated by the Association 


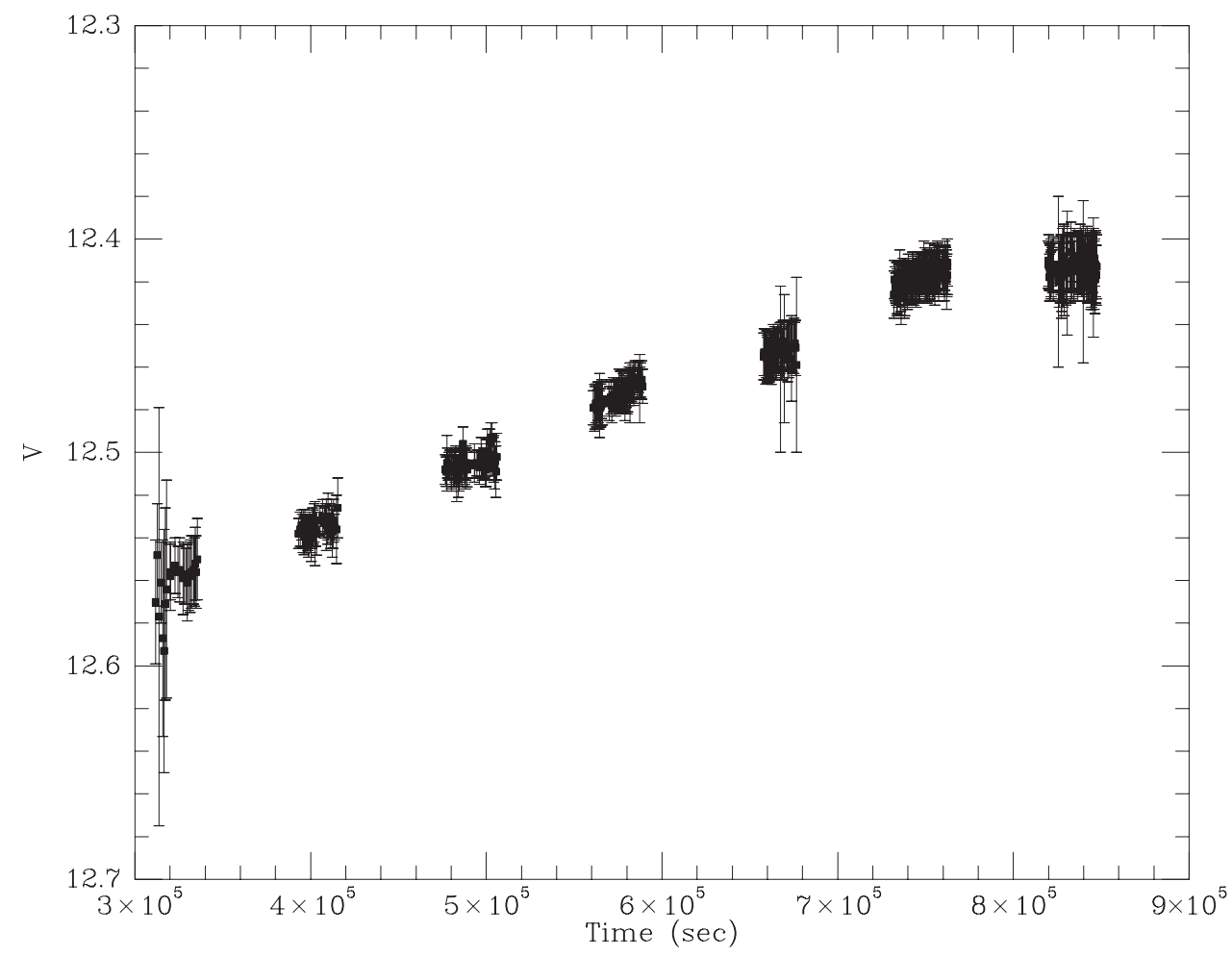

Figure 2 The optical lightcurve during 22-28 August 2000.

of Universities for Research in Astronomy, Inc. (AURA) under cooperative agreement with the National Science Foundation. SEST is operated by the European Southern Observatory and the Swedish National Facility for Radio Astronomy. This work was supported by the Centre of Excellence, and a Grant-in-Aid for Scientific Research by the Japan Ministry of Education, Science, Sports and Culture, and Australian Research Council.

\section{References}

Kardashev, N. S. 1962, SvA (AJ), 6, 317

Kataoka, J., et al. 2001, ApJ, 560, 659
Nishijima, K. 2002, PASA, 19, 26

Pacholczyk, A. G. 1970, Radio Astrophysics (San Francisco: Freeman)

Perlman, E. S., Madejski, G., Stocke, J. T., \& Rector, T. A. 1999, ApJ, 523, L11

Sambruna, R. M., Urry, C. M., Ghisellini, G., \& Maraschi, L. 1995, ApJ, 449, 567

Sambruna, R. M. 2000, GeV-TeV Gamma Ray Astrophysics Workshop: towards a major atmospheric Cherenkov detector VI, eds B. L. Dingus, M. H. Salamon, \& D. B. Kieda, AIP Conference Proceedings, 515, 19

Tagliaferri, G., et al. 2001, A\&A, 368, 38

Urry, C. M., Scarpa, R., O'Dowd, M., Falomo, R., Pesce, J. E., \& Treves, A. 2000, ApJ, 532, 816 